\title{
The accuracy of severity assessment tools designed for community acquired pneumonia among elderly patients with hospital acquired pneumonia
}

\author{
Doha Rasheedy \\ MD, Lecturer at Geriatrics and Gerontology Department, Faculty of Medicine, Ain Shams University, Cairo, Egypt
}

Background: Pneumonia severity assessment is an essential initial step in the evaluation of patients with community acquired pneumonia. Many validated severity assessment tools were designed for use in this setting. However, there is no disease based validated scoring system are in use for hospital acquired pneumonia. Aims and Objectives: The aim of this study was to assess the validity for the use of PSI, CURB-65, and SMARTCOP scores in elderly with hospital acquired pneumonia. Materials and Methods: Thirty patients with hospital acquired pneumonia and 41 patients with community acquired pneumonia were assessed using PSI, CURB-65, and SMART-COP scores. Short term outcomes were recorded i.e. in hospital mortality, or 30 days mortality and the need for mechanical ventilation or vasopressor drugs. Results: The 30 day mortality was $66.67 \%$ and $41.5 \%$ among HAP and CAP patients respectively. In both groups, the patients who died were older than the survivors; they had lower partial pressure of oxygen and Glasgow Coma Scale score. Moreover, they had higher heart rate and higher PSI, CURB 65, and SMART- COP scores. Among patient with community acquired pneumonia survivors had higher serum albumin while among patient with hospital acquired pneumonia, the survivors had lower fasting blood glucose. Conclusion: PSI predicted 30 day mortality and the use of mechanical ventilation but not the use of vasopressor drugs in hospital acquired pneumonia. SMART- COP and CURB 65 predicted the use of vasopressor drugs in this group.

Key words: Hospital acquired pneumonia, Pneumonia severity assessment scores, PSI, CURB 65, SMART- COP

\section{INTRODUCTION}

Pneumonia in elderly is a major public health problem causing significant morbidity and mortality. It represents the fifth leading cause of death in the elderly. ${ }^{1}$ The increased incidence and severity of pneumonia among this age group is attributed to immunosenescence and underlying comorbid medical disorders. ${ }^{2}$

Furthermore, hospitalization itself is a strong predictor for pneumonia as the second most common nosocomial infection; with the risk increases proportional to the length of hospital stay. ${ }^{3}$
According to the setting of onset, pneumonia is classified into community acquired (CAP) or nosocomial pneumonia (NP). The Nosocomial pneumonia subset is further classified into ventilator-associated pneumonia or hospitalacquired pneumonia (HAP). ${ }^{4}$

Different clinical guidelines agreed that the severity assessment is a mandatory initial step in the evaluation of patients presenting with CAP to guide treatment. ${ }^{5,6}$ The early identification of high risk patients is essential to plan care in advance, ensure patient safety and properly allocate the critical care resources. ${ }^{7}$ The most commonly used validated scores for determining the severity of CAP include the

Address for Correspondence:

Doha Rasheedy, MD, Lecturer at Geriatrics and Gerontology Department, Faculty of Medicine, Ain Shams University, Cairo, Egypt.

E-mail: doharasheedy@yahoo.com. Phone: +20-01001193544, Fax: 0020222664946 (C) Copyright AJMS 
Pneumonia Severity Index (PSI), ${ }^{8}$ the Confusion, Urea, Respiratory rate, Blood pressure, age $\geq 65$ (CURB-65), ${ }^{9}$ and the Systolic blood pressure, Multilobar infiltrates, Albumin, Respiratory rate, Tachycardia, Confusion, Oxygen and $\mathrm{pH}$ $(\text { SMART-COP })^{10}$ scores.

Although, there are many assessment tools designed for risk stratification in CAP setting, there is no agreement on the optimum one. ${ }^{11}$ Each score has its advantages and disadvantages but none of them was validated for use in HAP setting. Moreover, there is no other disease based validated scoring system in use for HAP. Thus, the patient cohorts recruited for HAP clinical trials are very heterogenous regarding mortality risk. This is a major limitation preventing proper randomization. ${ }^{12}$ Lack of sufficient validity for the severity scores of HAP in elderly, limits their use in clinical and research settings.

The aim of this study was to compare the performance of the different pneumonia severity scores for predicting 30 days mortality, the use of vasopressors and the need for mechanical ventilation among elderly patients in both community and hospital acquired pneumonia groups.

\section{PATIENTS AND METHODS}

A case-control study was conducted in the out-patient as well as in-patient of the Geriatrics Department at Ain Shams University Hospital, Cairo, Egypt over a period of twelve months from August 2014 to July 2015.

Seventy one patients aged 60 years and older were recruited and subdivided into group (1) that included 41 participants suffering from CAP and group (2) including 30 participants suffering from HAP.

CAP was defined according to the criteria proposed by Fang et al. ${ }^{13}:$ a) the presence of infiltrate in the admission chest $\mathrm{x}$-ray; b) the presence of one or more major criteria (cough, mucopurulent or hemoptic expectoration, temperature $>37.8{ }^{\circ} \mathrm{C}$ measured axillary); or c) at least two minor criteria (pleuritic chest pain, dyspnea, decreased level of consciousness, lung tissue consolidation detected by physical examination, or total leucocytes count $\geq 12000 / \mathrm{ml}$ ). While, HAP was defined as pneumonia that occurred 48 hours or more after hospital admission and not incubating at the time of admission. ${ }^{14}$

Exclusion criteria were pulmonary fibrosis, other infections e.g. urinary tract infection, skin and soft tissue infections etc, and malignancy.

At the time of initial evaluation, all participants were subjected to clinical assessment that included detailed personal and medical history, physical examination, and chest radiograph (postero-anterior and lateral views). Blood samples were collected for estimation of total leucocytes count, hemoglobin concentration, hematocrit, platelet count, fasting blood glucose, serum levels of Blood urea Nitrogen, Creatinine, Sodium, Potassium, Calcium, Phosphorus, Magnesium, total proteins, albumin, transaminases, and bilirubin. Arterial blood gases (ABGs) were also performed. Coexisting illnesses were diagnosed based on patients' self reports, data from medical record and the results of the previous investigations. ${ }^{15}$

Each patient was assessed for pneumonia severity using PSI, CURB-65, and SMART-COP scores.

PSI composed of 20 different parameters that include: age, sex, nursing home living, coexisting illnesses, physical examination findings, several laboratory, blood gas, and radiographic patterns. It is a five class scoring system stratified according to the increased mortality risk. ${ }^{7}$

The CURB 65 index includes confusion, urea nitrogen $(>7 \mathrm{mmol} / \mathrm{l})$, respiratory rate $(>30 / \mathrm{min})$, and blood pressure (diastolic pressure $<60 \mathrm{~mm} \mathrm{Hg}$ or systolic blood pressure of $<90 \mathrm{~mm} \mathrm{Hg})^{8}$

SMART-COP was designed to predict the need for intensive respiratory or vasopressor support (IRVS) among hospitalized patients with CAP. Criteria included were systolic BP $<90 \mathrm{mmHg}$, Multilobar Chest X-ray involvement, Albumin $<35 \mathrm{~g} /$ l, Respiratory rate $\geq 30 \mathrm{br} / \mathrm{min}$ in age above 50 , Tachycardia $\geq 125$ b.p.m, new onset confusion, Oxygenation $\leq 60 \mathrm{mmHg}$ or $\mathrm{O}_{2}$ sat $\leq 90 \%$ in age above 50, and $\mathrm{PH}<7.35$. $^{9}$

Finally, the clinical outcomes i.e. hospital discharge, in hospital mortality, or 30 days mortality were recorded. The need for mechanical ventilation or vasopressor drugs were recorded.

\section{Ethical consideration}

Informed consent was taken from every elder participating in this study or his surrogate. The study methodology was approved by the Research Review Board of the Geriatrics and Gerontology Department, Faculty of medicine, Ain Shams University.

\section{Statistical methods}

The data were tabulated and statistical analyzed using SPSS program (version 16). Quantitative variables were presented in the form of means and standard deviation. Qualitative variables were presented in form of frequency tables. The comparison between quantitative variables was done using $\mathrm{t}$ test. Comparison between qualitative variables was done 
using Pearson's Chi square test. Statistical difference was accepted when $\mathrm{p}<0.05$. Receiver operating characteristic (ROC) curves were constructed. The area under each ROC curve was calculated to assess the ability of the assessed score to predict recorded outcomes.

\section{RESULTS}

A comparison between cases with CAP and cases with HAP regarding their demographic characteristics are shown in Table 1. Both were matched for age and sex. Among patients with CAP the most common comorbidity was diabetes mellitus, followed by hypertension and Ischemic heart disease. Among the patients with HAP, the most common comorbidity was diabetes mellitus, followed by hypertension and cerebrovascular accidents.

Regarding the recorded outcomes, there was a significant difference between the two groups regarding 30 day mortality $(p=0.031)$. Twenty patients with HAP $(66.67 \%)$ died within 30 days [10 (33.3\%) of them died in hospital]. While, among cases with CAP seventeen (41.5\%) patients died within 30 days [10 (24.39\%) of them died in hospital]. Moreover, there was a significant difference between the

\begin{tabular}{|c|c|c|c|}
\hline Variable & $\begin{array}{l}\text { CAP } \\
\mathrm{N}=41\end{array}$ & $\begin{array}{l}\text { HAP } \\
N=30\end{array}$ & $P$ value \\
\hline Age & $69.63 \pm 7.61$ & $69.93 \pm 7.41$ & 0.475 \\
\hline \multicolumn{4}{|l|}{ Gender (\%) } \\
\hline Male & $20(48.8)$ & $17(56.7)$ & 0.339 \\
\hline Female & $21(51.2)$ & $13(43.3)$ & \\
\hline \multicolumn{4}{|l|}{30 days mortality (\%) } \\
\hline Survived & $24(58.5)$ & $10(33.33)$ & $0.031^{*}$ \\
\hline Died & $17(41.5)$ & $20(66.67)$ & \\
\hline \multicolumn{4}{|l|}{$\begin{array}{l}\text { Use of mechanical } \\
\text { ventilation }(\%)\end{array}$} \\
\hline Yes & $16(39.0)$ & $16(53.3)$ & 0.17 \\
\hline No & $25(61.0)$ & $14(46.7)$ & \\
\hline \multicolumn{4}{|l|}{ Use of vasopressor drug (\%) } \\
\hline Yes & $3(7.3)$ & $8(26.7)$ & $0.029^{*}$ \\
\hline No & $38(92.7)$ & $22(73.3)$ & \\
\hline PSI & $111.68 \pm 33.23$ & $135.10 \pm 29.49$ & $0.003^{*}$ \\
\hline CURB 65 & $2.07 \pm 1.00$ & $3.03 \pm 1.09$ & $0.000^{*}$ \\
\hline SMART- COP & $4.60 \pm 2.30$ & $6.13 \pm 2.57$ & $0.012^{*}$ \\
\hline \multicolumn{4}{|l|}{ Co existing illnesses (\%) } \\
\hline Diabetes mellitus & $25(61.0)$ & $18(60.0)$ & 0.563 \\
\hline Hypertension & $22(53.7)$ & $16(53.3)$ & 0.584 \\
\hline Ischemic heart disease & $14(34.1)$ & $9(30.0)$ & 0.457 \\
\hline Congestive heart failure & $7(17.1)$ & $5(16.7)$ & 0.612 \\
\hline $\begin{array}{l}\text { Chronic obstructive } \\
\text { pulmonary disease }\end{array}$ & $8(19.5)$ & $5(16.7)$ & 0.506 \\
\hline Bronchial asthma & $6(14.6)$ & $0(0)$ & $0.031^{*}$ \\
\hline Cerebrovascular accidents & $7(17.1)$ & $13(43.3)$ & $0.015^{*}$ \\
\hline Chronic kidney disease & $11(26.8)$ & $8(26.7)$ & 0.603 \\
\hline Chronic liver disease & $5(12.2)$ & $3(10.0)$ & 0.542 \\
\hline Smoking & $10(24.39)$ & $11(36.67)$ & 0.67 \\
\hline
\end{tabular}

two groups regarding the use of vasopressor drugs but not regarding the use of mechanical ventilation. The three studied severity assessment scores were significantly higher among patients with HAP compared to those with CAP (Table 1).

Among the CAP group, the patients who died were older than the survivors; they had lower partial pressure of oxygen, serum albumin level, and score of Glasgow Coma Scale. Moreover, they had higher heart rate and higher PSI, CURB 65, and SMART- COP scores. Similarly, among the HAP group, the patients who died were older than the survivors; they had lower partial pressure of oxygen and score of Glasgow Coma Scale and higher heart rate, fasting blood glucose and higher PSI and SMART- COP scores. As regards the effect of comorbidity, the coexisting illnesses showed no significant difference between survivors and non survivors in both groups (Table 2).

Figures 1,2, and 3 showed that, among CAP group, PSI had an area under the curve of 0.714 (95\% CI; 0.556- 0.873, $\left.\mathrm{p}=0.021^{*}\right)$ for predicting 30 day mortality, had area under the curve equal to 0.670 ( $95 \% \mathrm{CI}$; 0.505- $0.835, \mathrm{p}=0.044 *)$ for predicting the use of mechanical ventilation, and it was insignificant for predicting the use of vasopressor drugs. Similarly, SMART- COP had an area under the curve of 0.751 (95\% CI; 0.602- 0.901, $\left.\mathrm{p}=0.007^{*}\right)$ for predicting 30 day mortality, had area under the curve equal to 0.819 ( $95 \% \mathrm{CI}$; 0.682- 0.955, $\mathrm{p}=0.001 *)$ for predicting the use of mechanical ventilation, had area under the curve equal to 0.956 (95\% CI; 0.889- 1.023, $\mathrm{p}=0.009 *)$ for predicting the use of vasopressor drugs. CURB 65 had an area under the curve of 0.668 (95\% CI; 0.497- 0.839, $\mathrm{p}=0.05^{*}$ ) for predicting 30 day mortality, but it was insignificant for predicting the use of mechanical ventilation or the use of vasopressor drugs.

Among HAP group, PSI was the only tool with the ability to predict 30 day mortality, the use of mechanical ventilation but not the use of vasopressor drugs, while only SMART-COP and CURB 65 predicted the use of vasopressor drugs in this group.

Among HAP group, PSI had an area under the curve of 0.791 (95\% CI; 0.625- 0.959, $\mathrm{p}=0.011 *$ ) for predicting 30 day mortality, had area under the curve equal to $0.790(95 \% \mathrm{CI}$; 0.626- 0.955, $\mathrm{p}=0.007 *)$ for predicting the use of mechanical ventilation, and it was insignificant for predicting the use of vasopressor drugs (Figures 1-3). For predicting the use of vasopressor drugs among HAP group, CURB 65 had area under the curve equal to $0.889(95 \%$ CI; 0.713-1.056, $\left.\mathrm{p}=0.001^{*}\right)$, and SMART- COP had area under the curve equal to 0.929 (95\% CI; 0.827- 1.031, $\left.\mathrm{p}=0.000^{*}\right)($ Figure 3). 
Table 2: Comparison of clinical variables between survivors and patients who died in the two groups

\begin{tabular}{|c|c|c|c|c|c|c|}
\hline \multirow[t]{2}{*}{ Variables } & \multicolumn{2}{|c|}{ CAP } & \multirow[t]{2}{*}{$P$ value } & \multicolumn{2}{|c|}{ HAP } & \multirow[t]{2}{*}{$P$ value } \\
\hline & Survived & Dead & & Survived & Dead & \\
\hline Age & $67.00 \pm 3.70$ & $72.52 \pm 2.82$ & $0.04^{*}$ & $67.50 \pm 4.13$ & $77.56 \pm 5.47$ & $0.01^{*}$ \\
\hline TLC & $14.21 \pm 7.49$ & $16.14 \pm 8.43$ & 0.445 & $14.36 \pm 8.44$ & $13.28 \pm 6.17$ & 0.68 \\
\hline $\mathrm{PH}$ & $7.38 \pm 0.084$ & $7.35 \pm 0.084$ & 0.288 & $7.37 \pm 0.06$ & $7.33 \pm 0.13$ & 0.41 \\
\hline $\mathrm{PO}_{2}$ & $61.41 \pm 13.40$ & $55.42 \pm 10.00$ & $0.000^{*}$ & $57.60 \pm 2.81$ & $41.40 \pm 5.77$ & $0.000^{*}$ \\
\hline FBG & $189.30 \pm 86.80$ & $164.70 \pm 72.46$ & 0.358 & $158.50 \pm 80.03$ & $208.60 \pm 82.59$ & $0.01^{*}$ \\
\hline BUN & $45.23 \pm 37.70$ & $41.28 \pm 32.10$ & 0.68 & $44.40 \pm 27.40$ & $54.25 \pm 42.19$ & 0.51 \\
\hline $\mathrm{Na}$ & $135.4 \pm 5.99$ & $136.00 \pm 6.92$ & 0.76 & $137.30 \pm 6.49$ & $138.00 \pm 12.26$ & 0.86 \\
\hline Albumin & $3.09 \pm 0.55$ & $2.66 \pm 0.730$ & $0.04^{*}$ & $2.88 \pm 0.63$ & $2.56 \pm 0.50$ & 0.16 \\
\hline GCS & $14.50 \pm 1.86$ & $10.31 \pm 4.50$ & $0.000^{*}$ & $12.40 \pm 4.03$ & $8.60 \pm 4.90$ & $0.04^{*}$ \\
\hline Pulse & $95.12 \pm 19.28$ & $121.00 \pm 11.03$ & $0.001^{*}$ & $95.30 \pm 14.77$ & $130.00 \pm 29.78$ & $0.000^{*}$ \\
\hline Systolic blood pressure & $133.04 \pm 31.48$ & $130.62 \pm 35.49$ & 0.82 & $124.00 \pm 22.70$ & $109.07 \pm 31.60$ & 0.19 \\
\hline Diastolic blood pressure & $81.58 \pm 19.39$ & $76.87 \pm 15.37$ & 0.42 & $76.55 \pm 13.34$ & $67.00 \pm 21.05$ & 0.25 \\
\hline Temperature & $37.80 \pm 0.72$ & $37.54 \pm 0.55$ & 0.23 & $37.90 \pm 0.94$ & $37.74 \pm 0.88$ & 0.54 \\
\hline Respiratory rate & $25.50 \pm 6.92$ & $25.60 \pm 5.85$ & 0.95 & $25.40 \pm 7.26$ & $30.10 \pm 8.22$ & 0.13 \\
\hline PSI & $104.58 \pm 35.87$ & $121.70 \pm 27.01$ & $0.04^{*}$ & $127.00 \pm 20.93$ & $142.70 \pm 30.62$ & $0.01^{*}$ \\
\hline CURB 65 & $1.79 \pm 0.931$ & $2.47 \pm 1.00$ & $0.032^{*}$ & $2.60 \pm 1.17$ & $3.25 \pm 1.01$ & 0.12 \\
\hline SMART- COP & $3.75 \pm 1.96$ & $5.82 \pm 2.24$ & $0.003^{*}$ & $4.90 \pm 2.60$ & $6.75 \pm 2.55$ & $0.05^{\star}$ \\
\hline \multicolumn{7}{|l|}{ Coexisting illness } \\
\hline Diabetes mellitus & $16(66.70 \%)$ & $9(52.90 \%)$ & 0.28 & $5(50.0 \%)$ & $13(65.0 \%)$ & 0.34 \\
\hline Hypertension & $14(58.30 \%)$ & $8(47.10 \%)$ & 0.34 & $4(40.0 \%)$ & $12(60.0 \%)$ & 0.25 \\
\hline Ischemic heart disease & $9(37.50 \%)$ & $5(29.40 \%)$ & 0.42 & $5(50.0 \%)$ & $4(20.0 \%)$ & 0.10 \\
\hline Congestive heart failure & $3(12.50 \%)$ & $4(23.50) \%$ & 0.34 & $3(15.0 \%)$ & $2(10.0 \%)$ & 0.6 \\
\hline Chronic obstructive pulmonary disease & $6(25.00 \%)$ & $2(11.80 \%)$ & 0.26 & $2(20.0 \%)$ & $3(15.0 \%)$ & 0.51 \\
\hline Bronchial asthma & $4(16.70 \%)$ & $2(11.80 \%)$ & 0.51 & - & - & - \\
\hline Cerebrovascular accidents & $3(12.50 \%)$ & $4(23.50) \%$ & 0.34 & $5(50.0 \%)$ & $8(40.0 \%)$ & 0.446 \\
\hline Chronic Kidney disease & $8(33.30 \%)$ & $3(17.60 \%)$ & 0.22 & $5(50.0 \%)$ & $3(15.0 \%)$ & 0.056 \\
\hline Chronic Liver disease & $2(8.30 \%)$ & $3(17.60 \%)$ & 0.33 & $0(0 \%)$ & $3(15.0 \%)$ & 0.28 \\
\hline Smoking & $6(25.00 \%)$ & $4(23.50) \%$ & 0.42 & $5(50.0 \%)$ & $6(30.00 \%)$ & 0.34 \\
\hline
\end{tabular}

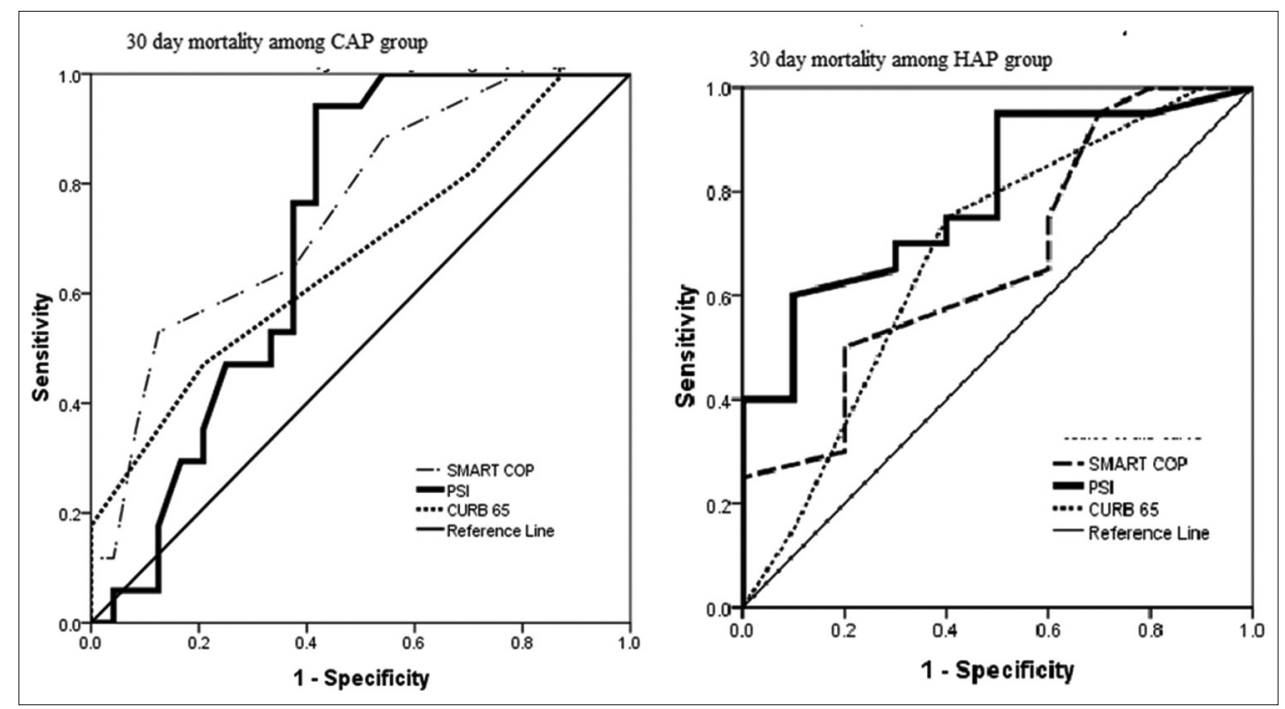

Figure 1: Receiver operating characteristic (ROC) curve for using PSI, SMART- COP and CURB 65 to predict 30 day mortality: The ROC curves show that the PSI, CURB 65 and SMART- COP scores had area under the curve of $0.714^{*}, 0.668^{*}$, and $0.751^{*}$ for predicting 30 day mortality in CAP group and had area under the curve of $0.790^{*}, 0.680$, and 0.668 for predicting 30 day mortality in HAP group, *Statistical significance

\section{DISCUSSION}

The aim of this study was to validate the use of PSI, CURB-65, and SMART-COP scores in HAP setting.
We examined the role of PSI, CURB-65, and SMARTCOP scores as predictors of 30-day mortality in patients admitted because of CAP or developed HAP during their course of hospital stay. The ability to predict the need for 


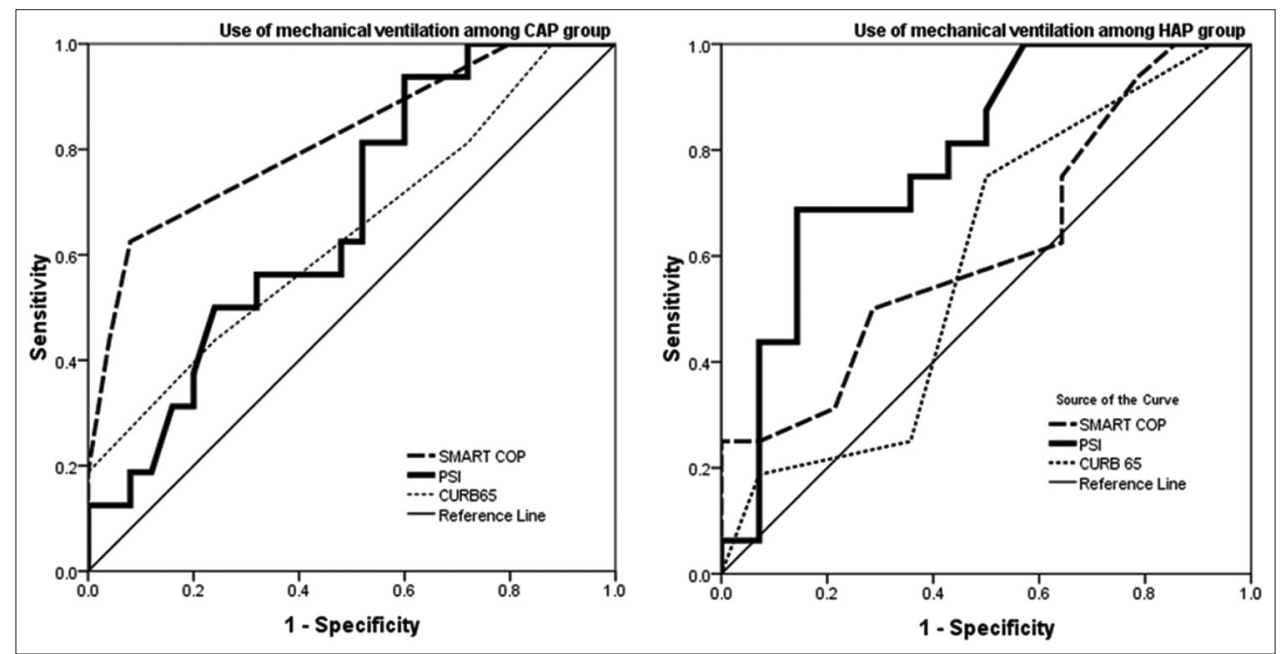

Figure 2: Receiver operating characteristic (ROC) curve for using PSI, SMART- COP and CURB 65 to predict the use of mechanical ventilation: The ROC curves show that the PSI, CURB 65 and SMART- COP scores had area under the curve of $0.670^{*}, 0.640$, and $0.819^{*}$ for predicting the use of mechanical ventilation in CAP group and had area under the curve of $0.790^{*}, 0.587$, and 0.621 for predicting the use of mechanical ventilation in HAP group, *Statistical significance
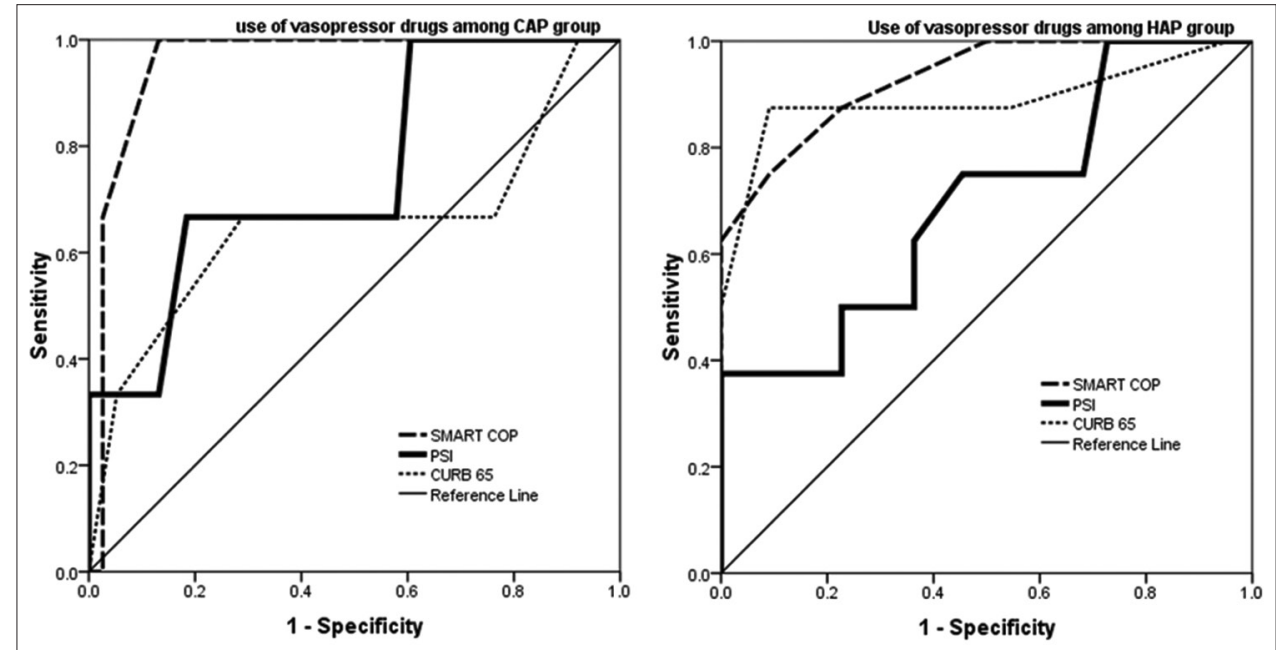

Figure 3: Receiver operating characteristic (ROC) curve for using PSI, SMART- COP and CURB 65 to predict the use of vasopressor drugs: The ROC curves show that the PSI, CURB 65 and SMART- COP scores had area under the curve of $0.750,0.654$, and $0.956^{*}$ for predicting the use of vasopressor drugs in CAP group and had area under the curve of $0.699,0.889^{*}$, and $0.929^{*}$ for predicting the use of vasopressor drugs in HAP group. *Statistical significance

intensive respiratory and vasopressor support was also evaluated.

Among the patient with CAP, seventeen (41.5\%) patients died within 30 days [10 (24.39\%) of them died in hospital] and the rest survived. This agrees with Calle et al. who reported in hospital mortality rate of $\left(24.2^{\circ}\right.$ ) during hospitalization. ${ }^{16}$ Previous data reported that CAP related mortality in elderly was $10 \%$ in hospital and $13 \%$ on follow up at 30 days. ${ }^{17}$

Lower mortality rate was reported by Ewing et al. ${ }^{18}$ it was $(13.72 \%$ and $14.44 \%)$ in 2005, 2006 respectively. This is attributed to inclusion of adults aged 18 years and older.
There was a statistically significant difference between survivors and patients who died in the CAP group regarding their age. This agrees with previous studies ${ }^{18-20}$ which reported that advanced age was a significant independent risk factor for mortality in CAP.

Among the CAP group, the patients who died had lower serum albumin level, and score of Glasgow Coma Scale. Moreover, they had higher heart rate. The same results were reported by Calle et al. ${ }^{16}$ Previous validation studies reported that CAP related mortality was associated with higher PSI, ${ }^{7}$ CURB $65,{ }^{8}$ and SMART- COP ${ }^{9}$ scores. Similar results were reported in the present study. 
Among the CAP group, the patients who died had lower partial pressure of oxygen. Previous results confirmed that hypoxemia was associated with higher mortality for hospitalized CAP in other studies. ${ }^{17,21}$

SMART-COP was the only score significant for predicting the use of vasopressor drugs in the CAP group $(\mathrm{AUC}=0.956)$ and it performed better than PSI in predicting 30 day mortality (AUC $=0.751$ and 0.714 respectively). Similarly, it performed better than PSI for predicting the use of mechanical ventilation (AUC $=0.819$ vs. 0.670 ).

In the SMART- COP validation study, ${ }^{10}$ the SMART- COP was better than PSI and CURB 65 in predicting the receipt of intensive respiratory or vasopressor support AUC $=0.87$, 0.69 , and 0.62 respectively.

According to Capelastegui et al. PSI had area under the curve (AUC) $0.888,95 \%$ confidence interval (CI) 0.864-0.912); while CURB-65 had (AUC 0.870, 95\% CI $0.844-0.895$ ) in predicting 30 -day mortality in $\mathrm{CAP}^{22}$

In the current study, twenty patients with HAP (66.67\%) died within 30 days with 10 (33.3\%) of them died in hospital. Previous data suggested that the in-hospital mortality in HAP was $51.5 \% .{ }^{23}$ According to Burton et al., mortality rate was $29 \%$ in patients with confirmed HAP, and $19 \%$ in patients with suspected $\mathrm{HAP}^{24}$

In the present study, only PSI had the prognostic power to predict 30 day mortality and the use of mechanical ventilation in the HAP group. Neither CURB-65 nor SMART-COP had the prognostic power to predict 30 day mortality or the use of mechanical ventilation in the HAP group.

Carrabba et al. reported that the 30-day mortality rate was $9.0 \%$ in the CAP group and $24.1 \%$ in the healthcare associated pneumonia (HCAP) group. In the HCAP group, the PSI had AUC of 0.68 and performed better than the CURB-65 score $(\mathrm{AUC}=0.62){ }^{25}$

This is in accordance with Fang et al., ${ }^{26}$ who demonstrated that PSI had moderate ability to discriminate survivors and the died patients at 30 days, and predicting the need for ICU admission. The PSI had the highest sensitivity to predicting mortality (AUC $=0.70$ ), followed by CURB-65 $(A U C=0.66)$. For predicting ICU admission on day 3 and day 14, the SMART-COP had AUC of 0.84 and 0.82 respectively.

Our study had several limitations; importantly this was a small single site observational study. With the small sample size, its statistical power might be insufficient.
In conclusion, this study demonstrated high 30 day mortality among elderly in both CAP and HAP groups. Among CAP group, the PSI, CURB 65, and SMART-COP scores allowed good discrimination for predicting 30 day mortality. Neither CURB-65 nor PSI allowed discrimination in predicting the use of vasopressor support. Among HAP group, the PSI allowed good discrimination for predicting 30 day mortality and the use of mechanical ventilation. Both CURB-65 and SMART- COP but not the PSI allowed the prediction of using vasopressor support in HAP group.

\section{ACKNOWLEDGMENTS}

The author acknowledge to the central laboratories in Ain Shams University.

\section{REFERENCES}

1. Lee $\mathrm{CH}$ and Wu CL. An Update on the Management of HospitalAcquired Pneumonia in the Elderly. International Journal of Gerontology 2008; 2 (4): 183-195.

2. Janssens JP and Krause KH. Pneumonia in the very old. The Lancet, Infectious Diseases 2004; 4:112-124.

3. Rotstein C, Evans G, Born A, Grossman R, Light RB, Magder S, et al. Clinical practice guidelines for hospital-acquired pneumonia and ventilator-associated pneumonia in adults. Can J Infect Dis Med Microbiol 2008; 19(1): 19-53.

4. Cascini S, Agabiti N, Incalzi RA, Pinnarelli L, Mayer F, Arcà M et al. Pneumonia burden in elderly patients: a classification algorithm using administrative data. BMC Infectious Diseases 2013; 13:559.

5. MacFarlane JT and Boldy JT. Update of BTS pneumonia guidelines: what's new? Thorax 2004; 59:364-366.

6. Mandell LA, Wunderink RG, Anzueto A, Bartlett JG, Campbell GD, Dean NC, et al. Infectious Disease Society of American/American Thoracic Society Consensus Guidelines for the Management of Community Acquired Pneumonia in Adults. Clin Infect Dis 2007; 44:S27-S72.

7. Marti C, Garin N, Grosgurin O, Poncet A, Combescure C, Carballo $S$ and Perrier A. Prediction of severe communityacquired pneumonia: A systematic review and meta-analysis. Critical Care 2012 16:R141.

8. Fine MJ, Auble TE, Yealy DM, Hanusa BH, Weissfeld LA, Singer DE, et al. A prediction rule to identify low-risk patients with community- acquired pneumonia. N Engl J Med 1997; 336:243-250.

9. Lim WS, van der Eerden MM, Laing R, Boersma WG, Karalus N, Town Gl, et al. Defining community acquired pneumonia severity on presentation to hospital: an international derivation and validation study. Thorax 2003; 58:377-382.

10. Charles PG, Wolfe R, Whitby M, Fine MJ, Fuller AJ, Stirling R, et al. SMART-COP: a tool for predicting the need for intensive respiratory or vasopressor support in community acquired pneumonia. Clin Infect Dis 2008; 47:375-384.

11. Singanayagam A, Chalmers JD and Hill AT. Severity assessment in community-acquired pneumonia: a review. Q J Med 2009; 102:379-388.

12. Napolitano LM. Use of Severity Scoring and Stratification Factors in Clinical Trials of Hospital-Acquired and Ventilator- Associated Pneumonia Clinical Infectious Diseases 2010; 51(S1):S67-S80. 
13. Fang GD, Fine $\mathrm{M}$ and Orloff $\mathrm{J}$. New and emerging etiologies for community-acquired pneumonia with implications for therapy. A prospective multicenter study of 359 cases. Medicine (Baltimore) 1990; 69:307-316.

14. American Thoracic Society and the Infectious Diseases Society of America. Guidelines for the management of adults with hospital-acquired, ventilator-associated, and healthcareassociated pneumonia. Am J Respir Crit Care Med 2005; 171:388-416.

15. Rasheedy D and Taha HM. Cardiac autonomic neuropathy: The hidden cardiovascular comorbidity in elderly patients with chronic obstructive pulmonary disease attending primary care settings. Geriatr Gerontol Int 2015; Feb 27. doi: 10.1111/ggi.12473. [Epub ahead of print]

16. Calle A, Márquez MA, Arellano M, Pérez LM, Pi-Figueras M and Miralles R. Geriatric assessment and prognostic factors of mortality in very elderly patients with community-acquired pneumonia. Arch Bronconeumol 2014; 50(10):429-434. doi: 10.1016/j.arbres.2014.01.012. Epub

17. Saldías Peñafiel F1, O'Brien Solar A, Gederlini Gollerino A, Farías Gontupil $\mathrm{G}$ and Díaz Fuenzalida A. Community-acquired pneumonia requiring hospitalization in immunocompetent elderly patients: clinical features, prognostic factors and treatment. Arch Bronconeumol 2003;39(8):333-340.

18. Ewig S, Birkner N, Strauss R, Schaefer E, Pauletzki J, Bischoff H, et al. New perspectives on community-acquired pneumonia in 388406 patients. Results from a nationwide mandatory performance measurement programme in healthcare quality. Thorax 2009; 64:1062-1069.

19. Tudose C, Moisoiu A and Bogdan M. Mortality Risk and Etiologic Spectrum of Community-acquired Pneumonia in Hospitalized
Adult Patients. Maedica (Buchar) 2010; 5(4): 258-264.

20. Klapdor B, Ewig S, Pletz MW, Rohde G, Schutte H, Schaberg T and Welte $T$ for the CAPNETZ study group. Communityacquired pneumonia in younger patients is an entity on its own. Eur Respir J 2012; 39: 1156-1161.

21. Sanz F, Restrepo MI, Fernandez E, Mortensen EM, Aguar MC, Cervera A, et al. Hypoxemia adds to the CURB-65 pneumonia severity score in hospitalized patients with mild pneumonia. Respir Care 2011; 56: 612-618.

22. Capelastegui A, Espana PP, Quintana JM, Areitio I, Gorordo I, Egurrola $\mathrm{M}$ and Bilbao $\mathrm{A}$. Validation of a predictive rule for the management of community-acquired pneumonia. Eur Respir $\mathrm{J}$ 2006; 27: 151-157.

23. Maruyama T, Niederman MS, Kobayashi T, Kobayashi $H$, Takagi T, D'Alessandro-Gabazza CN, et al. A prospective comparison of nursing home-acquired pneumonia with hospitalacquired pneumonia in non-intubated elderly. Respiratory 2008; 102 (9):1287-1295.

24. Burton LA, Price RJG, Barr KE, McAuley SM, Allen JB, Clinton A, et al. Incidence and Risk Factors For The Development Of Hospital Acquired Pneumonia In Older Hospitalised Patients. Thorax 2014; 69:A9-A10.

25. Carrabba M, Zarantonello M, Bonara P, Hu C, Minonzio F, Cortinovis I, et al. Severity assessment of healthcare-associated pneumonia and pneumonia in immunosuppression. Eur Respir J 2012; 40(5):1201-1210.

26. Fang WF, Yang KY, Wu CL, Yu CJ, Chen CW, Tu CY and Lin MC. Application and comparison of scoring indices to predict outcomes in patients with healthcare-associated pneumonia. Critical Care 2011; 15:R3.

Authors Contribution:

DR - Study design, acquisition, analysis and interpretation of data and, drafted the article.

Source of Support: The author received no financial support for the research or authorship of this article. Conflict of Interest: The author declared no conflicts of interest with respect to the authorship and publication of this article. 